\title{
POLINIZACIÓN DE DOS ESPECIES SIMPÁTRICAS DE STELIS (PLEUROTHALLIDINAE, ORCHIDACEAE)
}

\author{
Octavio Albores-Ortiz y Victoria Sosa* \\ Instituto de Ecología, A. C. Apartado Postal 63 \\ 91000 Xalapa, Veracruz, México \\ correo electrónico: victoria@ecologia.edu.mx
}

\begin{abstract}
RESUMEN
El objetivo de este trabajo es el de llevar a cabo estudios de polinización en las epífitas Stelis hymenantha y S. immersa que crecen simpátricamente en bosques de encino en la región de Ocosingo, Chiapas. Estas orquídeas pertenecen al linaje de las Pleurothallidinae, en el cual la polinización por dípteros es frecuente. Por medio de observaciones de campo en varias poblaciones de estos taxa se determinó su fenología, la situación de sus poblaciones, sus preferencias ecológicas, sus polinizadores así como su morfología floral y el tipo de atrayentes y recompensas que ofrecen. Los resultados indican que las flores de las dos especies coinciden en cierto momento en sus épocas de floración, que sus flores presentan grandes diferencias morfológicas y de tamaño, que atraen insectos al producir un líquido similar al néctar que se distribuye en forma de una capa delgada sobre la base del labelo y en una de las especies también en los pétalos, además de emitir fragancias diferentes. Stelis immersa recibe un mayor número de visitantes y sus polinizadores son hembras de un pequeño díptero, del género Megaselia (Phoridae). Aunque no se encontró un insecto llevando los polinarios de $S$. hymenantha, se observó a una mosca del grupo de los Drosophilidae penetrando las flores. La formación de cápsulas en las dos especies es muy baja en comparación con el número de flores. Una divergencia morfológica floral y una emisión de fragancias diferentes hacen que no se compartan los polinizadores, previniendo la hibridación entre estas orquídeas. Se requiere un tamaño específico de los polinizadores para que los polinarios sean removidos.
\end{abstract}

Palabras clave: Diptera, Drosophilidae, miofilia, néctar, Orchidaceae, Phoridae, polinización, Stelis.

\footnotetext{
*Autor responsable
} 


\section{ABSTRACT}

Stelis hymenantha and S. immersa are epiphytic orchids growing sympatrically on oaks in the area of Ocosingo, Chiapas. They belong to the group of Pleurothallidinae, in which pollination by Diptera is frequent. The objective of this paper is to determine pollination system as well as the mechanisms that prevent hybridization between these species. Field work was carried out in order to determine the number of individuals per population, flowering period, fruit set, as well as pollinators, attractants and rewards that the plants of these two species offer. Results indicate that S. hymenantha and S. immersa coincide in flowering during certain period of time, that the flowers have different size and morphology, that both produce fragrances, that are different, and that both produce a "nectarlike" transparent substance on the base of the lip and in one of the species also on the petals. Stelis immersa is pollinated by females of a fly in the genus Megaselia (Phoridae) and receives a large number of visitors. The pollinator of $S$. hymenantha was not determined precisely, however a Drosophilidae fly was seen entering flowers. Removal of pollinaria is determined by the size of pollinator. A very low number of capsules is produced in both species.

Key words: Diptera, Drosophilidae, Orchidaceae, Phoridae, Pleurothallidinae, pollination, Stelis.

\section{INTRODUCCIÓN}

Las Orchidaceae son las angiospermas en las que quizá se ha detectado la mayor diversidad de sistemas de polinización y polinizadores (Dodson, 1962; Pij1 y Dodson, 1969; Dafni, 1984; Ackerman, 1986; Tremblay, 1991; Nilsson, 1992; Johnson y Nilsson, 1999; Cozzolino y Windmer, 2005). Los insectos son los vectores más importantes en este grupo (Pijl y Dodson, 1969), siendo la miofilia -polinización por moscas- el segundo síndrome más común, con especies de 20 familias de dípteros registradas como polinizadoras (Christensen, 1994). Las flores emiten aromas que semejan el alimento natural o los substratos que visitan las moscas, los cuales comúnmente son materiales orgánicos en descomposición, excrementos o carroña, en búsqueda de alimento o para depositar sus huevecillos (Pijl y Dodson, 1969; Christensen, 1994). Diferentes partes florales producen olores putrescentes o dulces, comúnmente forman trampas que retienen al polinizador, además de poseer varios apéndices que los atraen y colores ya sea brillantes o que semejan carne o algún otro tinte de materia putrefacta (Chase, 1985; Johnson y Edwards, 2000; Borba et al., 2001a, b, 2002).

En las orquídeas, las barreras para prevenir la auto-polinización y la hibridización entre especies del mismo género por lo general existen a nivel de los mecanismos de la polinización (Pijl y Dodson, 1969; Dressler, 1993). Por ejemplo, 
una divergencia en el tiempo de floración previene el flujo de polen entre taxa simpátricos (Ellis y Johnson, 1999). Asimismo, el impedimento de intercambio polínico puede ser de carácter etológico, es decir que especies relacionadas presentan flores con morfología y fragancia distintas para atraer a diferentes polinizadores (Hills et al., 1972; Ellis y Johnson, 1999; Singer y Sazima, 1999, 2001a, b; Singer y Cocucci, 1999; Borba et al., 2001a, b).

Entre las Orchidaceae, uno de los grupos con más ejemplos de polinización por moscas es el de Pleurothallidinae, que es un linaje de pequeñas epífitas que se distribuye en las regiones intertropicales de América (Neyland, Urbatsch y Pridgeon, 1995; Luer, 2000; Pridgeon et al., 2001). Sus flores poseen caracteres de miofilia tales como forma radial de las mismas, labelo con manchas, pequeñas proyecciones, lóbulos fusionados, hendeduras y en algunos casos muy reducido (Pijl y Dodson, 1969; Christensen, 1994). Estudios en varios miembros de Pleurothallis, el género mejor conocido de las Pleurothallidinae, indican que los polinizadores son dípteros de las familias Chlorophidae y Phoridae, con una alta especificidad en las especies que visitan (Borba y Semir, 2001; Borba et al., 2001a, b, 2002).

Stelis es un género de las Pleurothallidinae con alrededor de 600 especies (Pridgeon y Chase, 2001), de las cuales 52 habitan en México, en muchos casos creciendo en forma simpátrica (Solano, 1993, 1999). En el único trabajo previo sobre autoecología de Stelis (S. argentata Lindl.) no se logró determinar su polinizador, aunque se observó actividad de moscas de la familia Drosophilidae (Christensen, 1992).

El objetivo de esta contribución es el de llevar a cabo estudios de ecología de polinización de dos especies de Stelis (S. hymenantha Schltr. y S. immersa (Linden \& Rchb. f.) Pridgeon \& M. W. Chase) que crecen simpátricamente en los bosques de encino de los alrededores de Ocosingo en Chiapas, para determinar si son miofílicas y cuáles son las barreras, si es que existen, que previenen la hibridación. Para cumplir con este objetivo, se llevaron a cabo observaciones en campo a fin de determinar su fenología, así como la situación de sus poblaciones, sus preferencias ecológicas, sus polinizadores y la morfología floral, así como el tipo de atrayentes y recompensas que ofrecen a los insectos.

\section{MATERIALES Y MÉTODOS}

Las poblaciones estudiadas crecen en el municipio de Ocosingo, que se encuentra al noreste de Chiapas. El área se localiza entre $16^{\circ} 50^{\prime}$ y $16^{\circ} 51^{\prime}$ de latitud norte y $92^{\circ} 06^{\prime}$ y $92^{\circ} 07^{\prime}$ de longitud oeste. La localidad se ve afectada por la convergencia entre dos tipos de clima: el cálido húmedo y el semicálido húmedo con abundantes lluvias en verano. La temperatura media anual es de alrededor de 
$24^{\circ} \mathrm{C}$ y la precipitación total anual es del orden de $1800 \mathrm{~mm}$. Las plantas se econtraron en un encinar formado por Quercus corrugata, Q. skinneri, Q. peduncularis, $Q$. polymorpha y $Q$. conspersa.

Se identificaron, cuantificaron y marcaron poblaciones de ambas especies (tres de Stelis hymenantha y ocho de S. immersa). Se contó el número de individuos juveniles y adultos, diferenciados los segundos por la presencia de inflorescencias o vestigios de éstas. Se observaron las condiciones físicas particulares en las que se desarrollan, como sustrato, orientación, altitud, y con un "data logger" se determinó la cantidad de luz que reciben. Se contó el número de inflorescencias, de flores y de cápsulas. Con ayuda de un microscopio de disección se midieron cada una de las estructuras florales. Se registró el tipo de apertura de las flores; los horarios de producción de atrayentes y recompensas y las estructuras donde se producen. Los ejemplares de respaldo se depositaron en los herbarios AMO, CORU y XAL. Para capturar los insectos se empleó una red entomológica y los especímenes fueron entregados a la colección IEXA. Se estableció un período de observación de entre seis y ocho horas (de las 7-8 de la mañana, a las 14-15 de la tarde) durante los meses de diciembre, enero y febrero de 2003, y febrero-marzo de 2004.

\section{RESULTADOS}

\section{Fenología}

Las plantas de Stelis hymenantha y S. immersa son epífitas que crecen sobre ramas de Quercus, y rara vez lo hacen sobre otros árboles. Ambas especies suelen encontrarse en el mismo huésped, así como presentar fenología semejante, pues florecen a principios de la temporada de invierno y producen similar número de flores por inflorescencia. Por su parte, la cantidad de cápsulas es reducida en las dos Stelis (Cuadros 1, 2, 3; Fig. 1).

\section{Morfología floral}

Stelis hymenantha y S. immersa tienen una morfología floral diferente (Fig. 2). S. hymenantha es una especie de plantas pequeñas, de hasta $18 \mathrm{~cm}$ de largo, que produce una o dos inflorescencias por tallo, hasta de $13 \mathrm{~cm}$ de largo. Las flores están dispuestas en dos hileras, orientadas hacia el mismo lado del racimo, son diminutas, atractivas durante el día, verdes o amarillo-verdosas, de alrededor de $2 \mathrm{~mm}$ de largo. Los sépalos están unidos en aproximadamente una quinta parte de su longitud, son cortamente apiculados y glabros en la superficie abaxial y glandulosos en la adaxial. El sépalo dorsal es ovado-elíptico, los laterales son 
Cuadro 1. Composición de las poblaciones estudiadas y la intensidad de luz que reciben.

\begin{tabular}{|c|c|c|c|c|c|c|c|c|c|c|c|c|}
\hline \multirow{2}{*}{\multicolumn{2}{|c|}{$\frac{\text { Especie }}{\text { Poblaciones }}$}} & \multicolumn{3}{|c|}{ Stelis hymenantha } & \multicolumn{8}{|c|}{ Stelis immersa } \\
\hline & & 1 & 2 & 3 & 1 & 2 & 3 & 4 & 5 & 6 & 7 & 8 \\
\hline \multirow{3}{*}{ 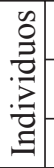 } & Adultos & 2 & 26 & 12 & 28 & 35 & 5 & 30 & 10 & 25 & 35 & 18 \\
\hline & Juveniles & 8 & 0 & 10 & 8 & 12 & 2 & 16 & 7 & 5 & 3 & 5 \\
\hline & Total & 10 & 26 & 22 & 36 & 47 & 7 & 46 & 17 & 30 & 38 & 23 \\
\hline \multicolumn{2}{|c|}{$\begin{array}{c}\text { Luz } \\
\text { (lumen/pie }{ }^{2} \text { ) }\end{array}$} & \multicolumn{3}{|c|}{$0.043-0.045$} & \multicolumn{4}{|c|}{$1.02-1.09$} & \multicolumn{4}{|c|}{$0.25-0.20$} \\
\hline
\end{tabular}

Cuadro 2. Floración y producción de atrayentes en las poblaciones estudiadas.

\begin{tabular}{|c|c|c|}
\hline Especies & Stelis hymenantha & Stelis immersa \\
\hline Época de floración & noviembre-enero & diciembre-marzo \\
\hline Número de inflorescencias & 30 & 66 \\
\hline Número de flores & 580 & 1496 \\
\hline Número de cápsulas & 15 & 57 \\
\hline Duración de la flor & 7-11 días & hasta 30 días \\
\hline Apertura de la flor & simultánea & simultánea \\
\hline $\begin{array}{l}\text { Horario de producción } \\
\text { de atrayentes }\end{array}$ & $8: 00$ - 14:00 hrs. & $7: 45-15: 40$ hrs. \\
\hline Horario de visitas & $8: 00-14: 00$ hrs. & $7: 30-15: 20$ hrs. \\
\hline $\begin{array}{l}\text { Estructuras en las que se } \\
\text { produce una sustancia } \\
\text { similar al néctar }\end{array}$ & labelo & $\begin{array}{c}\text { pétalos } \\
\mathrm{y} \\
\text { base del labelo }\end{array}$ \\
\hline
\end{tabular}

orbicular-ovados, de 0.5 a $0.9 \mathrm{~mm}$ de largo. Los pétalos miden 0.3 a $0.4 \mathrm{~mm}$ de largo y 0.2 a $0.3 \mathrm{~mm}$ de ancho, son erectos, largamente cuneado-obovados, con un nervio. El labelo, de 0.2 y $0.3 \mathrm{~mm}$ de largo, es subcuadrado a oblongo, incurvado en el ápice, con tres nervios, carnoso, con papilas, con dos callos en forma de semiluna, profundamente excavado al frente de los callos. La columna mide alrededor de $0.2 \mathrm{~mm}$ y está ensanchada hacia el ápice, el clinandrio es trilobado, la antera semiglobosa. El polinario está formado por dos polinios claviformes con caudícula y viscidio. El rostelo es saliente y laminar, el estigma está formado por dos lóbulos prominentes. 
Cuadro 3. Visitantes florales, polinizadores y conducta de los insectos.

\begin{tabular}{|c|c|c|}
\hline Especies & Stelis hymenantha & Stelis immersa \\
\hline Visitantes (Diptera) & $\begin{array}{l}\text { Drosophilidae (1 spp.) } \\
\text { Phoridae (1 spp.) } \\
\text { Una más no determinada }\end{array}$ & $\begin{array}{l}\text { Drosophilidae (2 spp.) } \\
\text { Muscidae (1 sp.) } \\
\text { Lonchaeidae (1 sp.) } \\
\text { Phoridae (6 spp.): } \\
\text { Dohrniphora sp. } \\
\text { Phalacrotophora sp. } \\
\text { Tachinidae (1 spp.) } \\
\text { Tres más no determinadas }\end{array}$ \\
\hline $\begin{array}{l}\text { Insectos de } \\
\text { otros grupos }\end{array}$ & & $\begin{array}{l}\text { Coleoptera: Curculionidae } \\
\text { Hymenoptera (2 spp.) } \\
\text { Hemiptera: Pyrrhocoridae: } \\
\text { Dysdercus sp. }\end{array}$ \\
\hline Polinizador & $\begin{array}{l}\text { No identificado con precisión } \\
\text { probablemente un díptero } \\
\text { de la familia Drosophilidae }\end{array}$ & $\begin{array}{l}\text { Megaselia (Phoridae) } \\
(1 \mathrm{sp} .)\end{array}$ \\
\hline $\begin{array}{l}\text { Conducta del } \\
\text { insecto }\end{array}$ & $\begin{array}{l}\text { La mosca vuela hacia la flor, } \\
\text { se posa sobre los sépalos y } \\
\text { acerca sus estructuras bucales } \\
\text { al centro (entre la columna y } \\
\text { el labelo); se adhiere o } \\
\text { deposita el polinario }\end{array}$ & $\begin{array}{l}\text { El insecto vuela cerca de la } \\
\text { planta; aterriza en alguna hoja, } \\
\text { permanece algunos minutos; } \\
\text { vuela hacia la flor y se queda } \\
\text { tomando o examinando la } \\
\text { substancia similar al néctar } \\
\text { que se produce en los pétalos } \\
\text { o introduce en la flor; es aqui } \\
\text { cuando se le adhiere o } \\
\text { deposita el polinario }\end{array}$ \\
\hline
\end{tabular}

Stelis immersa es una planta de hasta $17 \mathrm{~cm}$ de largo. Produce una sola inflorescencia, laxa y racemosa, de 15 a $20 \mathrm{~cm}$ de largo, cuya mitad se encuentra cubierta por la hoja. Los sépalos son carnosos, de color amarillento a algo naranjado o verde olivo, con flecos pequeños, densamente pubescentes por dentro y carinados al exterior, el sépalo dorsal obovado, cóncavo de 12 a $13 \mathrm{~mm}$ de largo y $3 \mathrm{~mm}$ de ancho; los sépalos laterales unidos casi hasta el ápice, formando una lámina cóncava, bífida, miden entre 11 y $13 \mathrm{~mm}$ de largo y $4 \mathrm{~mm}$ de ancho. Los pétalos son translúcidos, amarillento-anaranjados, espatulados, redondeados en el ápice, unguiculados, con un callo longitudinal que sobresale externamente a las venas, extendiéndose más allá del margen apical y variando entre 4 y $4.5 \mathrm{~mm}$ de largo. El labelo mide $4 \mathrm{~mm}$ de largo, grueso, carnoso, amarillento, con manchas púrpura-cafés, 


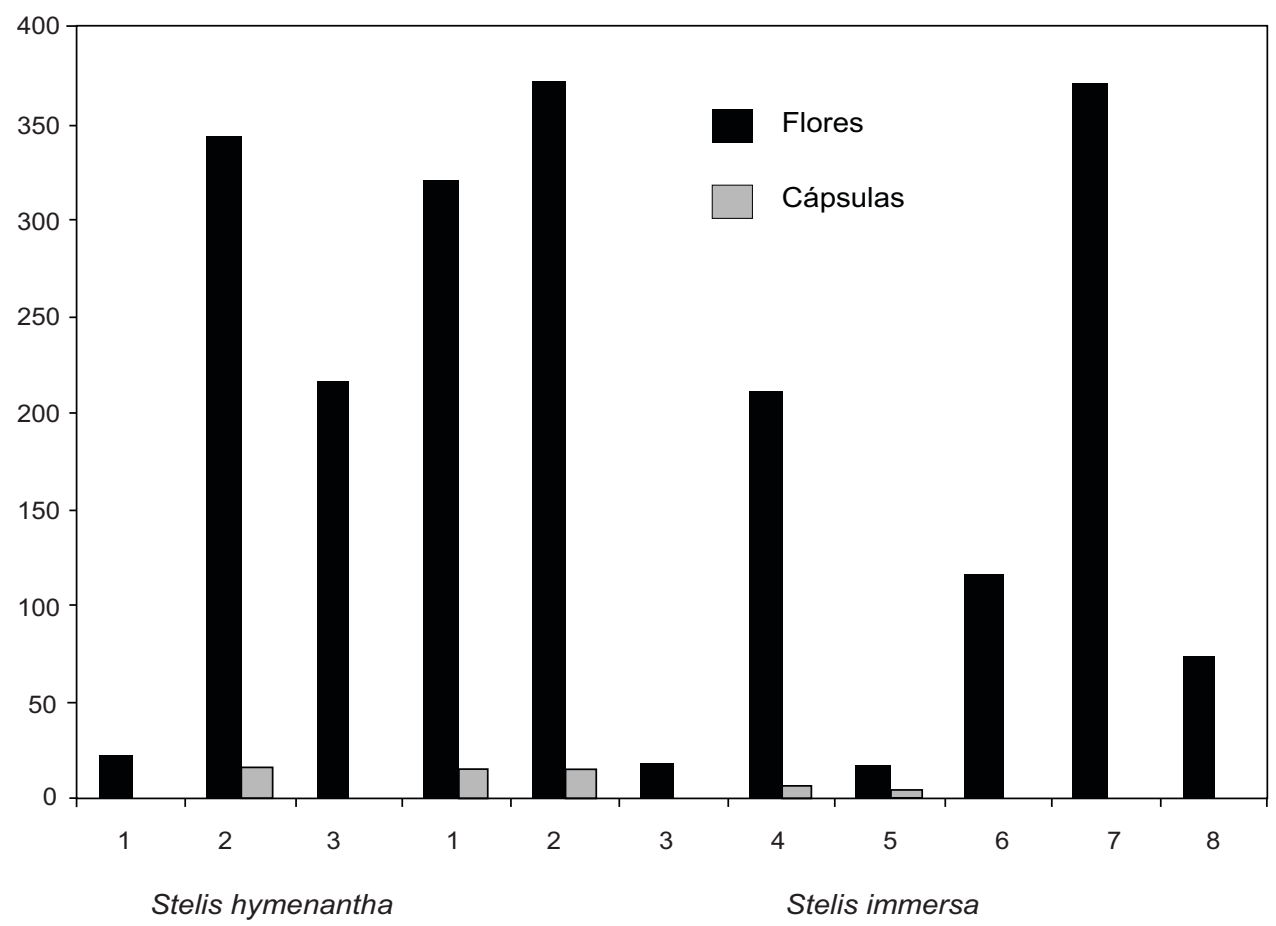

Fig. 1. Producción de flores y cápsulas en las poblaciones estudiadas de las dos especies de Stelis.

oblongo, obtuso hacia el ápice, unguiculado, los lados erectos con un callo por dentro en el margen, el disco engrosado, verrucoso y rugoso, bilobulado, articulado al pie de la columna. La columna tiene 4 a $5 \mathrm{~mm}$ de largo, es gruesa, alada longitudinalmente, con dientes y margen eroso hacia el ápice, la antera y estigma son ventrales.

Recompensas, polinizadores y visitantes

Las flores de Stelis hymenantha despiden un aroma intenso a mentol dulce. En la base del labelo se produce una substancia que se adhiere fuertemente a su superficie, a modo de película, semejante al néctar, imposible de medir por ser tan escasa. Las flores de $S$. immersa son también fragantes, con un aroma diferente, parecido al melón; pero a diferencia de la primera, la substancia pegajosa se localiza tanto en los pétalos como en la base del labelo.

Los visitantes de estas dos especies son principalmente dípteros de varias familias; compartiéndose algunas como Drosophilidae (Fig. 3) y Phoridae (Fig. 3, Cuadro 3). La mayoría de las moscas usualmente permanecen fuera de la flor "examinando" el líquido viscoso de la superficie externa de los pétalos. En 

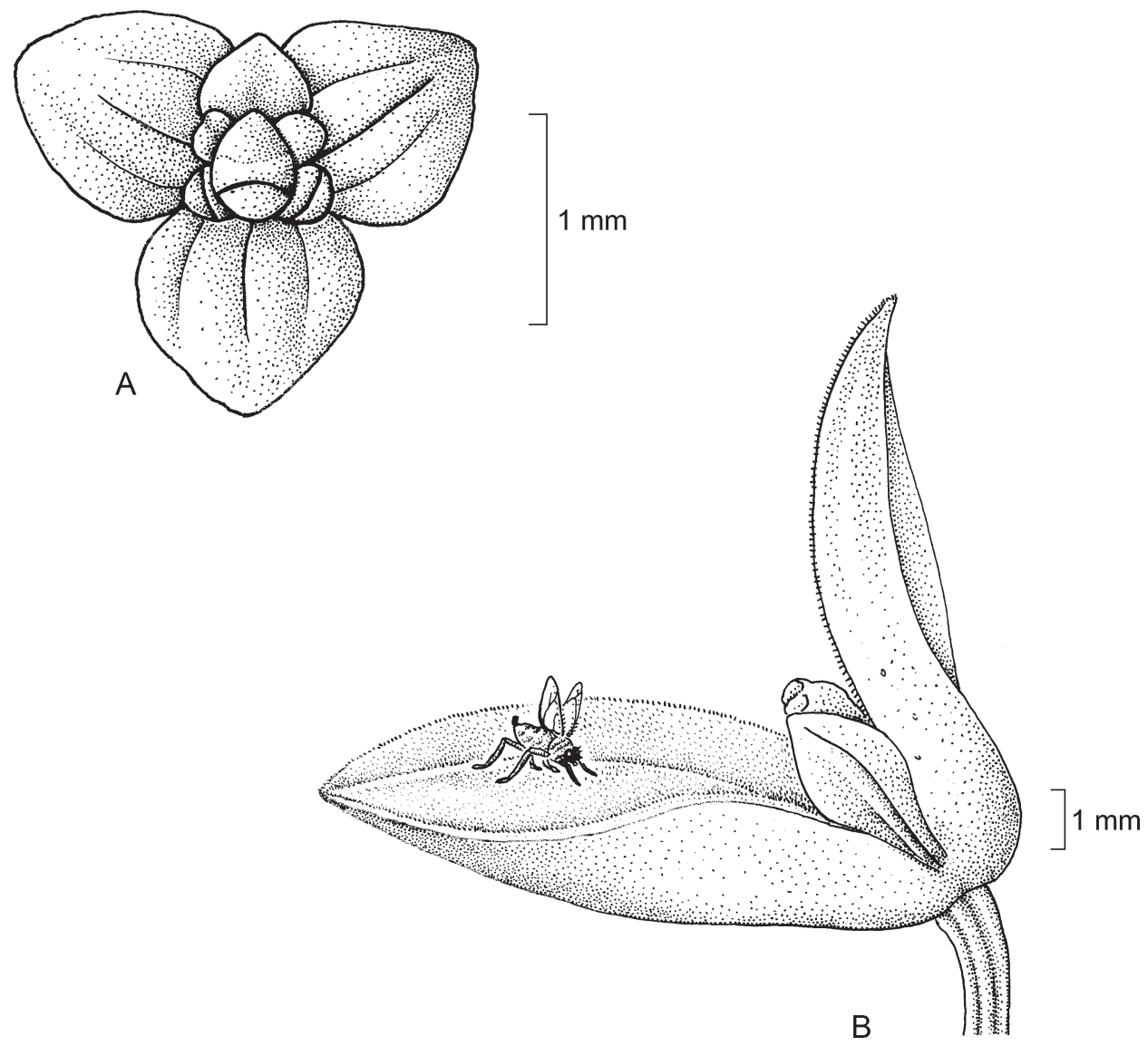

Fig. 2. Morfología floral. A. Stelis immersa. B. Stelis hymenantha.

ocasiones su gran tamaño les impide entrar a las flores. Además de las moscas, Stelis immersa es visitada por varios insectos de otros grupos (Cuadro 3), que al parecer también "examinan" esta substancia en las flores. Un coleóptero de los Curculionidae se alimenta de los fluídos de las flores de Stelis immersa, dañando severamente todas las estructuras.

Los polinizadores en Stelis immersa son hembras de una pequeña mosca del género Megaselia (Phoridae), la cual es la única con el tamaño preciso para efectuar la transferencia de polen. Después de examinar la substancia similar al néctar, el insecto se introduce lateralmente en la flor y se posa en el labelo que se encuentra en la posición dirigida hacia abajo. Cuando esto sucede, el labelo se levanta presionando al insecto contra el viscidio del polinario y atrapándolo. Para salir, la 

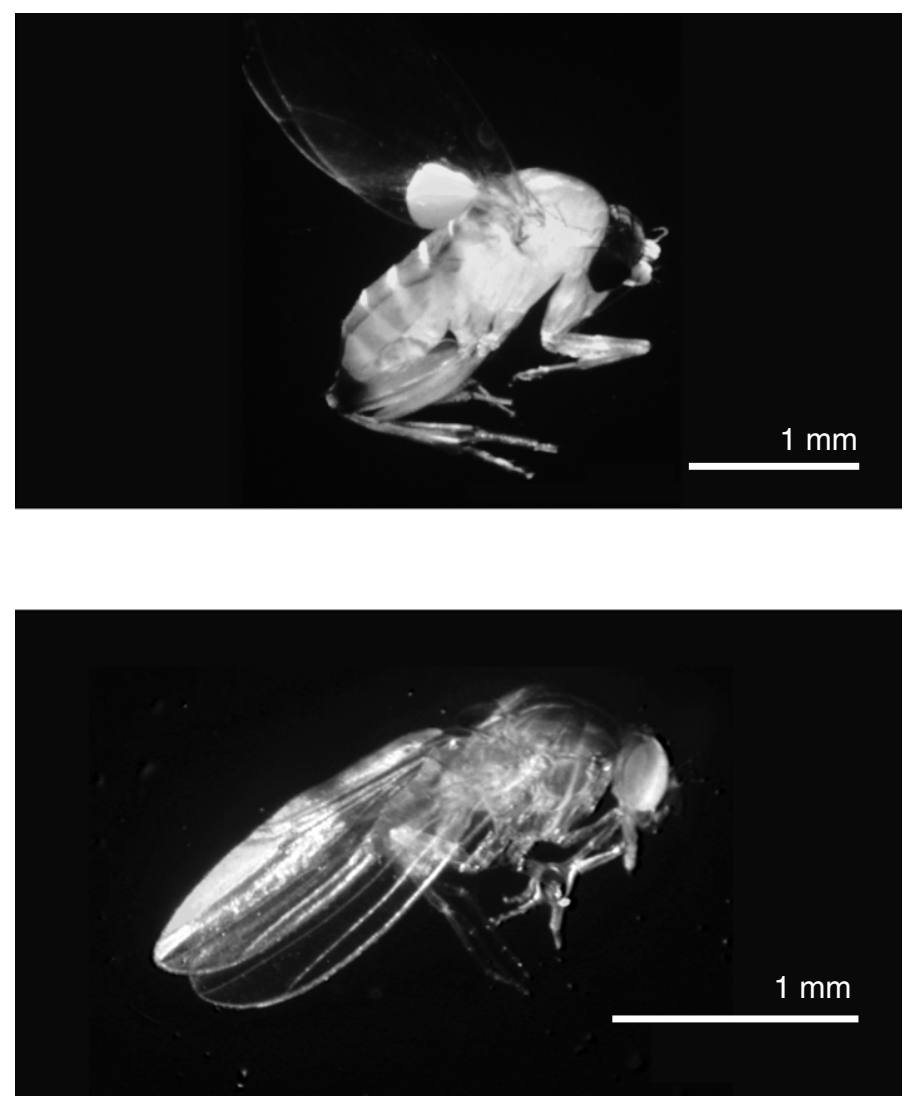

Fig. 3. Polinizadores y visitantes. A. Hembra de Megaselia sp. (Phoridae) con los polinarios de Stelis immersa adheridos en su tórax. B. Visitante de la familia Drosophilidae.

mosca retrocede y el viscidio se adhiere a su tórax. El labelo vuelve a su posición original liberando al animal. En el caso de $S$. hymenantha no se capturó a ningún díptero llevando polinarios, sin embargo, observamos a una mosca del grupo de las Drosophilidae entrando a las flores (Fig. 3).

\section{DISCUSIÓN}

El número de individuos por población de las dos especies estudiadas es similar, aunque en Stelis immersa es ligeramente mayor, alcanzando cerca de 50. Lo anterior coincide con lo conocido para otras Pleurothallidinae, como en el caso 
de Lepanthes caritensis, que tiene alrededor de 68 plantas por población (Tremblay et al., 1998). En algunos casos, especialmente en $S$. hymenantha, éstas carecen totalmente de individuos juveniles.

Aunque Stelis hymenantha inicia su período de floración más temprano, desde noviembre, coincide algún tiempo con el de $S$. immersa. La duración de las flores de la última es más prolongada, hasta 30 días, en comparación con S. hymenantha, en la cual éstas únicamente permanecen abiertas hasta once días. El horario en el que producen atrayentes y en el que son polinizadas y visitadas ambas orquídeas es muy semejante.

Las diferencias en morfología floral entre las dos especies de Stelis resultan en el aislamiento reproductivo, ya que no comparten polinizadores. Las flores de $S$. hymenantha son mucho más pequeñas que las de $S$. immersa. Más aún, en $S$. immersa tienen color que va de amarillento a anaranjado y están llenas de pelos y flecos que se mueven con el aire, señales precisas para atraer a dípteros, a su vez la forma campanulada del perianto se debe a la fusión de los sépalos laterales. En contraste, las de $S$. hymenantha son más amarillentas en el centro, el labelo es más obscuro y en general presentan menos estructuras móviles, siendo abierta la disposición de sépalos y pétalos.

La morfología floral divergente en las dos Stelis estudiadas se encuentra vinculada con el criterio de circunscripción genérica de Pridgeon y Chase (2001), que seguimos en este trabajo. Los mencionados autores transfirieron a Stelis a un buen número de especies de varias secciones de Pleurothallis, además de algunas de otros géneros, como consecuencia de resultados del análisis filogenético basado en secuencias de ADN. Stelis immersa fue una de las especies que se trasladaron de Pleurothallis. Con estos cambios, Stelis sufrió una expansión, incluyendo a plantas con gran diversidad morfológica.

La producción de fragancias también favoreció el aislamiento al atraer a diferentes grupos de dípteros. Stelis hymenantha produce un aroma similar al mentol, el cual es dulce y penetrante, en tanto que $S$. immersa tiene un aroma más discreto, similar al del melón. En la literatura se ha señalado el papel que juega la divergencia de fragancias en la atracción de polinizadores. Así, de acuerdo con Hills et al. (1972), tan sólo un cambio en los componentes de los aromas emitidos por flores de varias especies de Catasetum logra atraer a insectos totalmente diferentes.

La producción de un líquido similar al néctar que forma una capa delgada en la superficie del labelo y de los pétalos en las dos Stelis estudiadas, ya había sido señalada por Borba y Semir (2001) y Borba et al. (2001b, 2002) para varias Pleurothallidinae. Más aún, al analizar ciertas especies de Stelis, se descubrió que en los pétalos y en los labelos de algunos taxa, se producen cristales de oxalato de calcio, que funcionan como pseudonéctar para atraer a moscas (Chase y Peacor, 1987). También se encontró que las Stelis que producen un líquido similar al néctar 
no tienen pseudonéctar (Chase y Peacor, 1987); tal y como sucede en las Stelis observadas en este estudio, que carecen de estos cristales.

Los polinizadores y visitantes florales observados en nuestras Stelis son dípteros que previamente se conocían en relación con otras orquídeas. Por ejemplo, el vector de Pleurothallis teres es una especie de Megaselia (Phoridae) (Borba y Semir, 2001). Otros Phoridae se han registrado vinculados con algunas Rhizanthella (Christensen, 1994). Entre los Drosophilidae, Drosophila immigrans se ha encontrado como agente transportador del polen de Pleurothallis endostachys (Chase, 1985).

El gran número de flores, el tiempo que permanecen abiertas, los atrayentes ofrecidos y el elevado número de visitantes y polinizadores registrado en este estudio, harían suponer un alto porcentaje de fecundación. Sin embargo, los resultados muestran que apenas se forman algunos frutos. Ni aun en Stelis immersa, en la cual las flores permanencen abiertas por más tiempo, se desarrolla un mayor número de cápsulas. En las Orchidaceae se ha sugerido que la baja producción de frutos indica una limitación de polinizadores (por ej. Ackerman y Montalvo, 1990). Quizá en el caso de las dos especies de Stelis estudiadas se pueda argumentar la misma hipótesis, la cual se probaría con una colecta exhaustiva de los insectos del área en la época de floración.

Otra explicación de la baja producción de cápsulas que se ha propuesto en orquídeas es el comportamiento de sus polinizadores. Si los insectos no vuelan ciertas distancias, visitando diferentes plantas y poblaciones y si las especies son autoincompatibles, los polinarios pueden ser depositados en las flores de los mismos individuos, sin lograr éxito reproductivo (Johnson y Nilsson, 1999). Un ejemplo de lo anterior ha sido registrado en Bulbophyllum (Borba et al., 1999). Para probar esta hipótesis sería necesario determinar si las dos especies estudiadas son autoincompatibles, así como las distancias que pueden recorrer los dípteros.

Un aspecto notable es que en Stelis immersa la substancia similar al néctar se produce en la superficie externa de los pétalos y en la base del labelo, mientras este último está elevado hacia la columna, evitando que insectos muy grandes puedan penetrar a las flores y quedar atrapados. Las pequeñas hembras del Megaselia que polinizaron exitosamente a esta especie son de tamaño adecuado, examinaron el líquido similar al néctar, entraron a las flores, quedaron atrapadas $\mathrm{y}$ al salir se adhirieron los polinarios a su tórax.

\section{AGRADECIMIENTOS}

El primer autor de este trabajo agradece al Instituto de Ecología, A. C. una beca de licenciatura, para llevar a cabo este trabajo. También agradece a la 
Universidad Veracruzana, a la Facultad de Ciencias Biológicas y Agropecuarias, zona Orizaba-Córdoba las facilidades prestadas. Los autores agradecen a los Drs. Vicente Hernández y Luis Cervantes, de la colección IEXA, la identificación de algunas especies de polinizadores y visitantes, así como las facilidades otorgadas para el manejo de los ejemplares entomológicos; al Dr. Rodolfo Solano la identificación de los individuos de Stelis, así como al Dr. Brian Brown la de los dípteros de la famila Phoridae; al Biól. Rolando Jiménez Machorro su ayuda en la obtención de referencias bibliográficas; al Biól. Manuel Escamilla la elaboración de las ilustraciones. Agradecemos al Consejo Nacional de Ciencia y Tecnología su apoyo (P39601526) para desarrollar este proyecto.

\section{LITERATURA CITADA}

Ackerman, J. D. 1986. Mechanisms and evolution of food-deceptive pollination systems in orchids. Lindleyana 1: 108-113.

Ackerman, J. D. y A. M. Montalvo. 1990. Short- and long-term limitations to fruit production in a tropical orchid. Ecology 71: 263-271.

Borba, E. L., G. J. Shepherd y J. Semir. 1999. Reproductive systems and crossing potential in three species of Bulbophyllum (Orchidaceae) occurring in Brazilian "campo rupestre" vegetation. Pl. Syst. Evol. 217: 205-214.

Borba, E. L., J. M. Felix, V. N. Solferini y J. Semir. 2001b. Fly-pollinated Pleurothallis (Orchidaceae) species have high phenetic variability: evidence from isozyme markers. Am. J. Bot. 88: 419-428.

Borba, E. L. y J. Semir. 2001. Pollinator specificity and convergence in fly pollinated Pleurothallis (Orchidaceae) species: a multiple population approach. Ann. Bot. 88: 75-88.

Borba, E. L., J. Semir y G. J. Shepherd. 2001a. Self-incompatibility, inbreeding depression and crossing potential in five Brazilian Pleurothallis (Orchidaceae) species. Ann. Bot. 88: 89-99.

Borba, E. L., G. J. Shepherd y C. van der Berg. 2002. Floral and vegetative morphometrics of five Pleurothallis (Orchidaceae) species: correlation with taxonomy, phylogeny, genetic variability and pollination systems. Ann. Bot. 90: 219-230.

Chase, M. W. 1985. Pollination of Pleurothallis endosthachys. Am. Orch. Soc. Bull. 54: 431-434.

Chase, M. W. y D. M. Peacor. 1987. Crystals of calcium oxalate hydrate on the perianth of Stelis Sw. Lindleyana 2: 91-94.

Christensen, D. E. 1992. Notes on the reproductive biology of Stelis argentata Lindl. (Orchidaceae: Pleurothallidinae) in eastern Ecuador. Lindleyana 7: 28-33.

Christensen, D. E. 1994. Fly pollination in the Orchidaceae. In: Arditti, J. (ed.). Orchid biology: reviews and perspectives. VI. John Wiley \& Sons. Ithaca. New York. pp. 415-454. 
Cozzolino, S. y A. Windmer. 2005. Orchid diversity: an evolutionary consequence of deception? TREE 20: 487-494.

Dafni, A. 1984. Mimicry and deception in pollination. Ann. Rev. Ecol. Syst. 15: 259-278.

Dodson, C. H. 1962. The importance of pollination in the evolution of the orchids of tropical America. Am. Orch. Soc. Bull. 31: 525-534, 641-649, 731-735.

Dressler, R. L. 1993. Phylogeny and classification of the orchid family. Dioscorides Press, Portland, Oregon. 312 pp.

Ellis, A. G., y S. D. Johnson. 1999. Do pollinators determine hybridization patterns in sympatric Satyrium (Orchidaceae) species? Pl. Syst. Evol. 219: 137-150.

Hills, H. G., H. W. Norris y C. H. Dodson. 1972. Floral fragrances and isolating mechanisms in the genus Catasetum (Orchidaceae). Biotropica 4: 61-76.

Johnson, S. D. y L. A. Nilsson. 1999. Pollen carryover, geitonogamy, and the evolution of deceptive pollination systems in orchids. Ecology. 80: 2607-2619.

Johnson, S. D. y T. J. Edwards. 2000. The structure and function of orchid pollinaria. Pl. Syst. Evol. 222: 243-269.

Luer, C. A. 2000. Systematics of Jostia, Andinia, Barbosella, Barbrodria, Pleurothallis, subgen. Antilla, subgen. Effusia, subgen. Restrepioidia, addenda to Lepanthes, Masdevallia, Platystele, Pleurothallis, Restrepiopsis, Scaphosepalum and Teagueia. Icones Pleurothallidinarum 20. Missouri Botanical Garden, St. Louis. pp. 3-4.

Neyland, R., L. E. Urbatsch y A. M. Pridgeon. 1995. A phylogenetic analysis of subtribe Pleurothallidinae (Orchidaceae). Bot. J. Linn. Soc. 117: 13-28.

Nilsson, L. A. 1992. Orchid pollination biology. Trends Ecol. Evol. 7: 255-259.

Pijl, L. van der y C. H. Dodson. 1969. Orchid flowers. Their pollination and evolution. University of Miami Press. Coral Gables. pp. 101-122.

Pridgeon, A. M. y M. W. Chase. 2001. A phylogenetic reclassification of Pleurothallidinae (Orchidaceae). Lindleyana 16: 235-271.

Pridgeon, A. M., R. R. Solano y M. W. Chase. 2001. Phylogenetic relationships in Pleurothallidinae (Orchidaceae): combined evidence from nuclear and plastid DNA sequences. Am. J. Bot. 88: 2286-2308.

Singer, R. B. y A. A. Cocucci. 1999. Pollination mechanisms in four sympatric southern Brazilian Epidendroideae orchids. Lindeyana 14: 47-56.

Singer, R. B. y M. Sazima. 1999. The pollination mechanism in the "Pelexia alliance" (Orchidaceae: Spiranthinae). Bot. J. Linn. Soc. 131: 249-262.

Singer, R. B. y M. Sazima. 2001a. The pollination mechanism of three sympatric Prescottia (Orchidaceae: Prescottinae) species in southeastern Brazil. Ann. Bot. 88: 989-997.

Singer, R. B. y M. Sazima. 2001b. Flower morphology and pollination mechanism in three sympatric Goodyerinae orchids from southeastern Brazil. Ann. Bot. 88: 989-997.

Solano, R. 1993. El género Stelis en México. Orquídea (Mex.) 13: 1-75.

Solano, R. 1999. Orchidaceae III. Stelis. Flora de Veracruz. Fasc. 113. Instituto de Ecología, A. C. Xalapa, Veracruz. pp. 2-3.

Tremblay. R. L. 1991. Trends of pollination ecology of the Orchidaceae: evolution and systematics. Can. J. Bot. 70: 624-650. 
Acta Botanica Mexicana 74: 155-168 (2006)

Tremblay, R. L., J. K. Zimmerman, L. Lebron, P. Bayman, I. Sastre, F. Axelrod y J. AlersGarcia. 1998. Host specificity and low reproductive success in the rare endemic Puerto Rican orchid Lepanthes caritensis. Biol. Cons. 85: 297-304.

Recibido en septiembre de 2004.

Aceptado en noviembre de 2005. 